\begin{tabular}{ccc}
\hline & International Journal of Engineering \& Technology, $7(2.14)(2018) 114-118$ \\
SPC & International Journal of Engineering \& Technology \\
Website: $w w w . s c i e n c e p u b c o . c o m / i n d e x . p h p / I J E T$ & Research Paper \\
\hline
\end{tabular}

\title{
Filling Sharp Features on Corner of Triangular Mesh by using Enhanced Advancing Front Mesh (EAFM) method
}

\author{
Noorehan Awang ${ }^{1,2}$, Rahmita Wirza Rahmat ${ }^{1 *}$, Puteri Suhaiza Sulaiman', \\ Azmi Jaafar ${ }^{1}$, Ng Seng Beng ${ }^{1}$ \\ ${ }^{1}$ Faculty of Computer Science and Information Technology, Universiti Putra Malaysia, Serdang, Selangor. \\ ${ }^{2}$ Faculty of Computer and Mathematical Sciences, Universiti Teknologi MARA, Malaysia. \\ *Corresponding author E-mail:rahmita@fsktm.upm.edu.my
}

\begin{abstract}
Repairing an incomplete polygon mesh constitutes a primary difficulty in 3D model construction, especially in the computer graphics area. The objective of hole-filling methods is to keep surfaces smoothly and continually filled at hole boundaries while conforming with the shapes. The Advancing Front Mesh (AFM) method was normally used to fill simple holes. However, there has not been much implementation of AFM in handling sharp features. In this paper, we use an AFM method to fill a holes on sharp features. The Enhanced Advancing Front Mesh (EAFM) method was introduced when there was a conflict during triangle creation. The results of the study show that the presented method can effectively improve the AFM method, while preserving the geometric features and details of the original mesh.
\end{abstract}

Keywords: Meshes, Holes, Corner, Features, Boundary

\section{Introduction}

3D measurement approaches have evolved rapidly; the acquisition of the triangular-mesh model of an actual object no longer presents a particular challenge. Due to factors in scanning, light reflections, model shapes, contact areas of models, and other aspects, parts that cannot be captured may result, leading to partial 3D data that produces holes in point cloud models. Because of this problem, numerous post-processing methods have to be applied to an unprocessed model before it can be used as input for animation design applications. In computer graphics, repairing incomplete polygon meshes can still pose a basic challenge in 3D model construction. Hole-filling methods comprise some of the primary factors.

Hole-filling methods continually and smoothly fill surfaces at hole boundaries in conformance with the shapes. Hole-filling ideally offers the following features: (1) Robustness, or the ability to cover arbitrary holes for all models (robustness), (2) Efficiency, or the ability to fill large holes in realistic amounts of time, and (3) Precision, which enables patched surfaces to complement missing geometries. But because of hole complexity and variety, no one existing technique for filling holes satisfies every preferred property. Robustness can be singularly difficult to attain, particularly with various hole types[1].

Holes are closed loops that have all feature a boundary edge. Many kinds of holes can be created on surfaces depending on the scanned data points. Normal holes can be considered as simple holes; however, some surfaces may also contain missing holes on sharp features such as edges or corners in CAD models. Recently, a lot of research, based on filling holes, has been developed on a 3D mesh [2-6].
The AFM method was normally used to fill simple holes; however, there are not many implementations of the AFM method in handling sharp features on corners. One research, based on the AFM method, introduced new rules for angle estimation. This was due to a problem during hole filling on closed mesh models. Qualitative and quantitative analyses show that this method can approximate the original missing meshes and transition smoothly with the surrounding meshes [7].Another research used the AFM method to refine the boundary edges of holes. During the refining process, small angles between adjacent boundary edges are removed and concave boundary edges become almost convex. Later, the Delaunay triangulation was applied to construct new meshes for filling holes [8]. Problems with holes on mesh surfaces have also been seen in plant organs; where researchers fill holes with new points using the AFM method. The calculations of the new points were highlighted with three different angle criteria. Later, the normal vector and the normal curvature were estimated after the process was done [9]. In other research, a topological framework, based on handle-body theory for AFM implementation was done. The object was triangulated using AFM and a Ball-pivoting algorithm was used according to its topological aspects[10].

Two studies have combined the AFM and Delaunay approaches. The initial attempt began by firstly discretising fronts through the addition of nodes in accordance with previously-specified element densities. Points with locations as circumscribed in Delaunay's approach are ascertained, while the optimal equilateral triangle is approximated[11].The results of the second semiconductor structure study also proved improved quality meshes compared to the Delaunay only grid [12].

Motivated by previous researchers, this paper presents an enhanced AFM method. First, test data surfaces were extracted to create holes. 
Next, the AFM method was used to fill the holes. Finally, the EAFM method was introduced when there was a conflict during triangle creation on the sharp features on the corner. The length and angle was used as a reference to check accuracy of the method. The method presented is able to effectively improve the AFM method while preserving the geometric features and details of the original mesh.

\section{Experimental Procedure}

In this work, we adopt the advancing front mesh (AFM) technique to generate an initial patch mesh over the hole [1]. The method consists of the following six steps:

Step 1 : Initialize the front using the boundary vertices of the hole. Step 2: Calculate the angle $\theta_{i}$ between two adjacent boundary edges $\left(e_{i-1}\right.$ and $\left.e_{i+1}\right)$ at each vertex $v_{i}$ on the front.

Step 3: Starting from the vertex $v_{i}$, new triangles was created according to three angle criteria. The angle criteria to create new triangle was defined by three points defined as points A,B and C as shown in Figure 1.

a)If $\theta \leq 75^{\circ}$,the new triangle was created by connecting point $\mathrm{A}$ and $\mathrm{C}$, thus $\triangle A B C$ is directly constructed.

b) If $75<\theta<135^{\circ}$,one new point (NP1) was created and it create two triangles.

c) If $\theta \geq 135^{\circ}$,two new points NP1 and NP2 was inserted and it create three triangles.

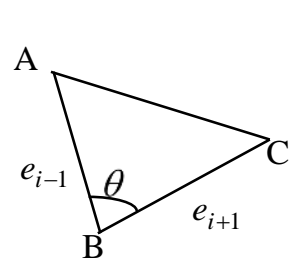

a)

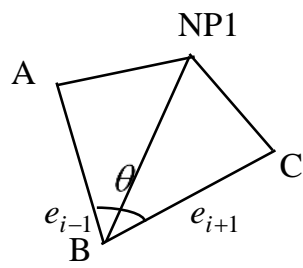

b)

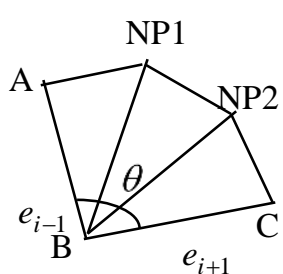

c)
Fig. 1: Three rules of angle in AFM

(a) $\theta \leq 75^{0}$ (b) $75^{0}<\theta<135^{\circ}$ (c) $\theta \geq 135^{\circ}$

Step 4: Compute the distance between each newly created vertex and every related boundary vertex. If the distance between them is less than the given threshold, they are merged as in (1)

$$
\text { Threshold }=\frac{1}{4} \times(\overline{N P 1 B} \text { or } \overline{N P 2 B})
$$

(1)

$(\overline{N P 1 B}$ or $\overline{N P 2 B}$ is the distance between new vertex and related boundary vertex).

Step 5: Update the front.

Step 6: Repeat step 1through step 4 until the whole region of holes has been patched by new triangle.

According to Table 1, 36 data points were used as test data for the enhanced method [13]. The data was used to test the sharp features on the corner. The initial stage of the hole-filling method requires the identification of existing holes in the triangular meshes. Next, the AFM method was used to fill the holes with triangles.
Table 1: 36 Data Points

\begin{tabular}{|c|c|c|c|c|c|}
\hline \multirow{2}{*}{ No } & \multicolumn{2}{|c|}{ Coordinates } & \multirow{2}{*}{ No } & \multicolumn{2}{|c|}{ Coordinates } \\
\hline & $\mathrm{X}$ & $\mathrm{y}$ & & $x$ & $\mathrm{y}$ \\
\hline 1 & 0.00 & 0.00 & 19 & 0.80 & 0.85 \\
\hline 2 & 0.50 & 0.00 & 20 & 0.85 & 0.65 \\
\hline 3 & 1.00 & 0.00 & 21 & 1.00 & 0.50 \\
\hline 4 & 0.15 & 0.15 & 22 & 1.00 & 1.00 \\
\hline 5 & 0.70 & 0.15 & 23 & 0.50 & 1.00 \\
\hline 6 & 0.50 & 0.20 & 24 & 0.10 & 0.85 \\
\hline 7 & 0.25 & 0.30 & 25 & 0.00 & 1.00 \\
\hline 8 & 0.40 & 0.30 & 26 & 0.25 & 0.00 \\
\hline 9 & 0.75 & 0.40 & 27 & 0.75 & 0.00 \\
\hline 10 & 0.85 & 0.25 & 28 & 0.25 & 1.00 \\
\hline 11 & 0.55 & 0.45 & 29 & 0.00 & 0.25 \\
\hline 12 & 0.00 & 0.50 & 30 & 0.75 & 1.00 \\
\hline 13 & 0.20 & 0.45 & 31 & 0.00 & 0.75 \\
\hline 14 & 0.45 & 0.55 & 32 & 1.00 & 0.25 \\
\hline 15 & 0.60 & 0.65 & 33 & 1.00 & 0.75 \\
\hline 16 & 0.25 & 0.70 & 34 & 0.19 & 0.19 \\
\hline 17 & 0.40 & 0.80 & 35 & 0.32 & 0.75 \\
\hline 18 & 0.65 & 0.75 & 36 & 0.79 & 0.46 \\
\hline
\end{tabular}

However, conflicts occurred in some cases during the creation of triangles, which caused the triangles to be unconnected. Figure 2 highlights problems when the AFM method was used to fill the holes on the sharp features on the corner. There occur some new points which is situated outside the boundary of holes thus the new point consider invalid. Therefore there is a need to enhance the method to handle such situations.

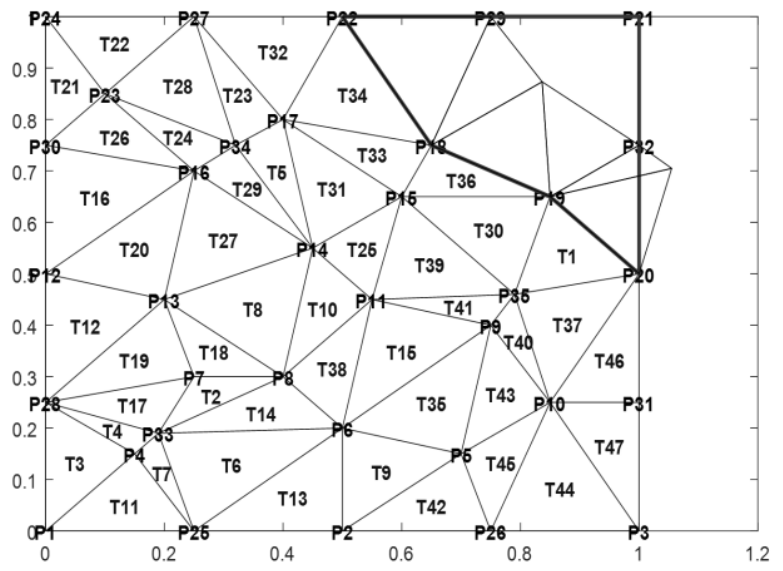

Fig. 2: Sharp Features problems

\section{Experimental Result}

\subsection{Normal Cases of AFM method}

Case 1

The three points were chosen as a starting point for triangle creation The law of cosines gives the value of the included angle $\theta$ when the lengths of two sides are known. For the AFM method, the included angle $\theta$ was used as a reference for selection of three different angle criteria. Figure 3 highlights the triangle obtained when creating a triangle on the corner of boundary holes. The first and second triangle was the triangle with angle $\theta \geq 135^{\circ}$ while the third triangles are using $\theta \leq 75^{\circ}$. 


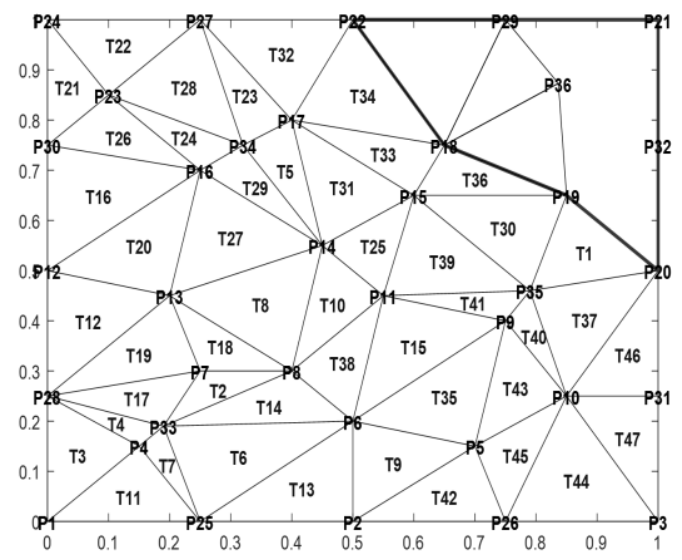

(a)

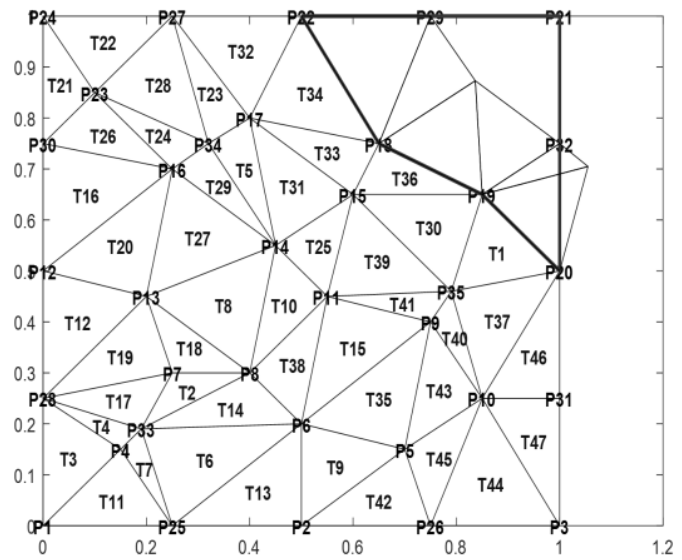

(b)

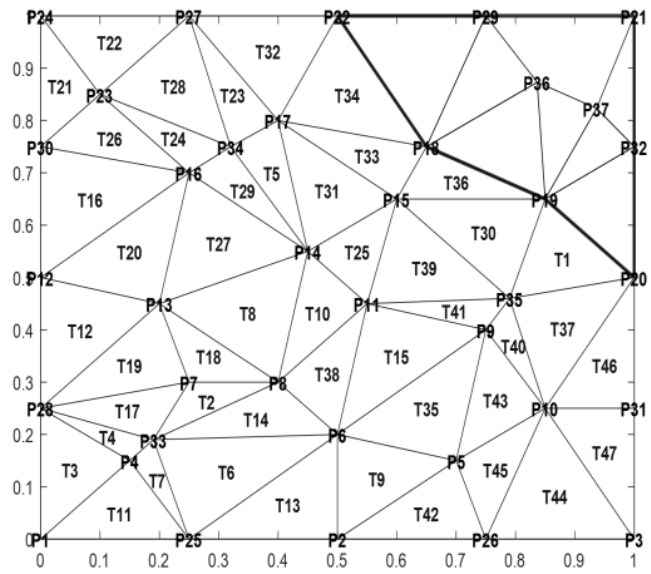

(c)

Fig. 3: Triangular element according to angle criteria

(a) $\theta \geq 135^{\circ}$ (b) $\theta \geq 135^{\circ}$ (c) $\theta \leq 75^{\circ}$

\section{Case 2}

The new points (NP) should be connected to any nearest active point, in order for the holes to be closed. The threshold value as in (1) was used as a reference. If there is an active point in range of the threshold, the active point replaces the position of the new point, and is defined as a new node. For example, in Figure 4, the creation of $\theta \geq 135^{0}$ requires two triangles to be constructed. There are two new points and the the point need to be check whether it is less than a given threshold value. Points P29 replaces the position of first new point since it was less than the threshold radius value. However, the second point (P36) remains constant as there is no nearest active node.

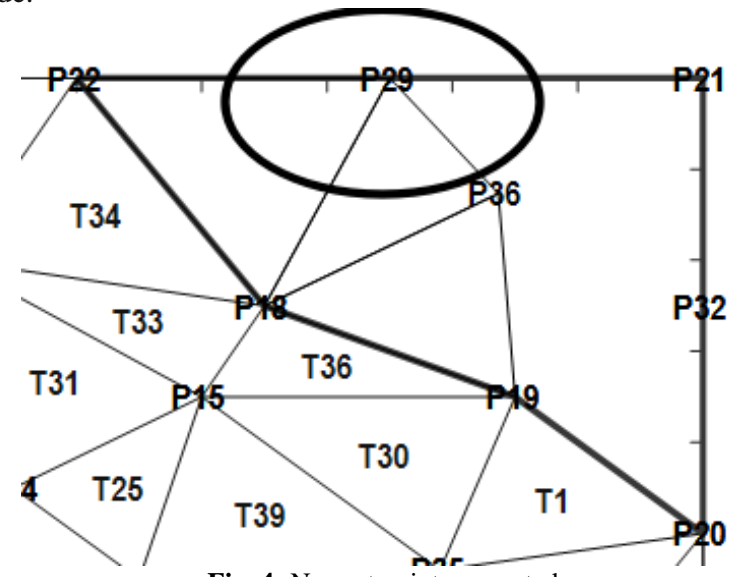

Fig. 4: Nearest point connected

\subsection{EAFM method}

\section{EAFM1}

Case 3

There are some cases where the distance between the new point and active nodes is greater than the given threshold. A circle with centre at the NP and radius $r$ given by the empirical formula [14] as in(2) where:

$\delta^{\prime}=\left\{\begin{array}{c}0.55 \times l \text { if } \delta<0.55 \times l \\ l \quad \text { if } 0.55 \times l \leq \delta \leq 2.0 \times l \\ 2.0 \times l \text { if } \delta>2.0 \times l\end{array}\right.$

Length $l$ was equal to distance of new vertex to point B while $r=0.6 \times \delta^{\prime}$

\section{Case 4}

Case 4 refers to a situation where, during triangle creation, three edges are automatically connected to themselves. This extension of cases was similar to the new cases use in EAFT-1 on the triangular mesh [15]. As shown in figure 5, Triangle 58(T58) was automatically constructed with three connected edges (known as P29, P36 and P37).

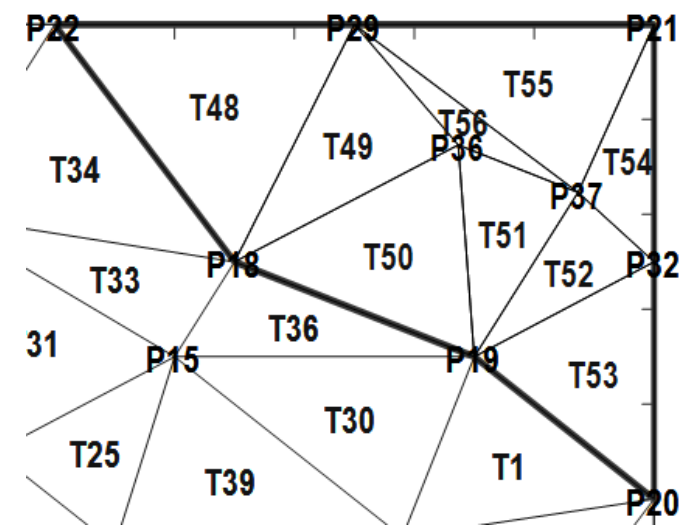

Fig. 5:Three edges connected

\section{EAFM2}

According to Figure 6, a problem occurs during triangle creation of third angle criteria for $\theta \geq 135^{\circ}$.During construction, two new points NP1 and NP2 was created and it create three triangles. However, the two new points was outside the boundary of holes. Therefore, the triangle cannot be considered valid and a new algorithm was introduced to the AFM method to make it applicable for such a situation 


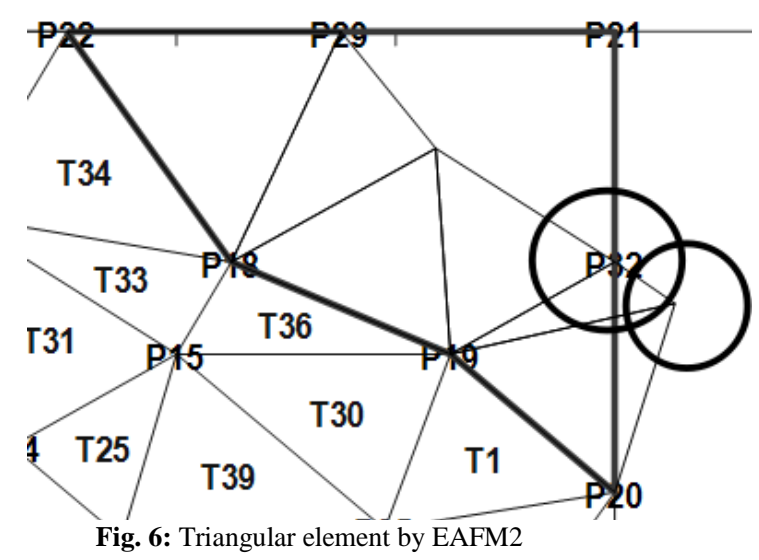

Therefore, a new algorithm, based on the midpoint formula, was used where the midpoint of $\mathrm{A}$ and $\mathrm{C}$ (point $\mathrm{M}$ ) was calculated in (3). $\mathrm{M}$ can also be considered as a perpendicular bisector between point $\mathrm{A}$ and $\mathrm{C}$. Then the same equation was applied to find NP1 and NP2 where it was a midpoint between AM and MC. Figure 7 illustrates the new algorithm.

$\operatorname{Midpoint}(\mathrm{M})=\left(\frac{A_{x}+C_{x}}{2}, \frac{A_{y}+C_{y}}{2}\right)$

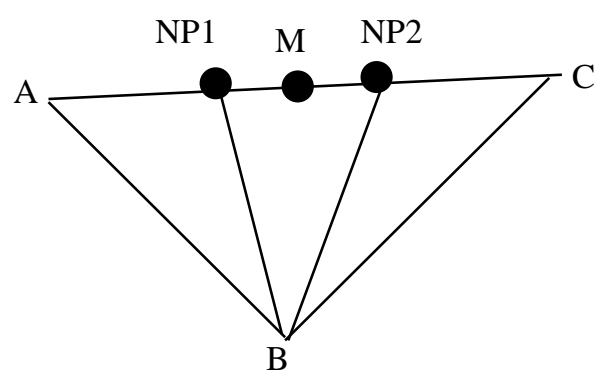

Fig. 7: EAFM2 algorithm

After trisecting triangle into three equal area, the searching of nearest node according to the case 2 of AFM method was applied. Figure 8 shows that point NP1 is equal to $\mathrm{P} 37$ while the second point was nearest to the active nodes of P32, thus NP2 gives a result of P32. Figure 9 shows the results of triangle creation after using the new algorithm.

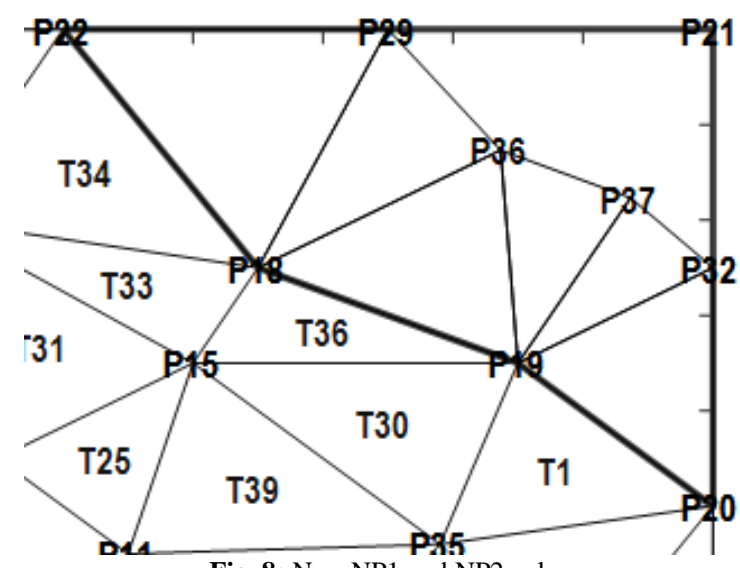

Fig. 8: New NP1 and NP2 value

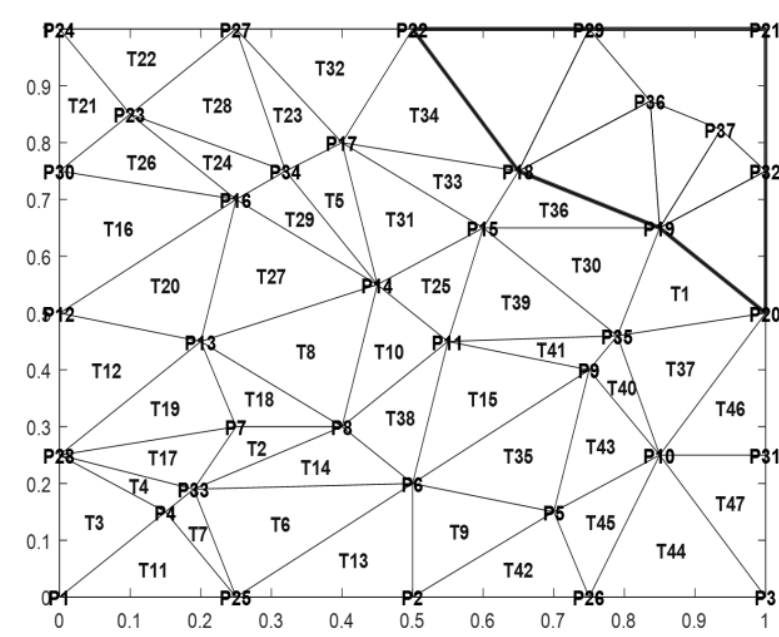

Fig. 9: Result after EAFM2

\subsection{Error Evaluation}

Table 2 shows the number of vertices of the filling patch and the number of triangles of the filling patch for small and big holes. The number of vertices increases after hole patching; since the method introduces new points to the holes; thus increasing the number of triangle creations.

Table 2: Vertices and triangle number before and after filling patch

\begin{tabular}{ccccc}
\hline \multirow{2}{*}{$\begin{array}{c}\text { Holes } \\
\text { data }\end{array}$} & \multicolumn{2}{c}{$\begin{array}{c}\text { Vertices number of fill- } \\
\text { ing patch }\end{array}$} & $\begin{array}{c}\text { Triangle number on filling } \\
\text { patch }\end{array}$ \\
\cline { 2 - 5 } & before & after & before & after \\
\hline Test data & 8 & 9 & 7 & 9
\end{tabular}

\section{Conclusion}

AFM method was normally used to fill simple holes. However, there has not been much implementation of AFM in handling sharp features. In this paper, 36 test data points was used to implement EAFM2 method in order to handle sharp features on corners. The method use midpoint formula to ensure the triangle was divided into equal area and lie inside the boundary. The results of the study shows that EAFM2 was able to solve problem related to sharp features on the corners and the method will be used later in a Real 3D object.

\section{Acknowledgement}

I would like to give my gratitude and thank you for Universiti Teknologi Mara (UiTM) and Kementerian Pelajaran Malaysia (KPM) scholarship for funding scholarship for the the journal publication.

\section{References}

[1] Zhao W, Gao S, \& Lin H (2007),A robust hole-filling algorithm for triangular mesh, The Visual Computer, 23(12),987-997.

[2] Fortes MA, González P, Palomares A \& Pasadas M (2017),Filling holes with geometric and volumetric constraints, Computers \& Mathematics with Applications.

[3] Cho JH, Song W, Choi H \& Kim T (2017), Hole Filling Method for Depth Image Based Rendering Based on Boundary Decision, IEEE Signal Processing Letters, 24(3),329-333.

[4] Chouvatut V \& Boonchieng E (2017),Brain tumor's approximate correspondence and area with interior holes filled, In Computer Science and Software Engineering (JCSSE), 2017 14th International Joint Conference on IEEE, July, pp. 1-5.

[5] Luo G, Zhu Y \& Guo B (2017), Fast MRF-based Hole Filling for View Synthesis, IEEE Signal Processing Letters. 
[6] Schmidt RM (2017),U.S. Patent No. 9,747,721. Washington, DC: U.S. Patent and Trademark Office.

[7] Zhang X, Duan L \& Xue T (2014), A high-quality closed model generation approach for triangular mesh,JOURNAL OF INFORMATION \& COMPUTATIONAL SCIENCE,11(10),3433-3440.

[8] Altantsetseg E, Matsuyama K \& Konno K (2015),Minimum Surface Area Based Complex Hole Filling Algorithm of 3D Mesh, The Journal of Art and Science, 14(2),26-35.

[9] Sun Z, Guo X, Lu S, Wen W \& Chen Y (2013), Filling holes in triangular meshes of plant organs, In International Conference on Computer and Computing Technologies in Agriculture,Springer, Berlin, Heidelberg, September, pp. 222-231.

[10] Medeiros E, Velho L \& Lopes H (2003), A topological framework for advancing front triangulation, In Computer Graphics and Image Processing, 2003. SIBGRAPI 2003, XVI Brazilian Symposium on IEEE, October, pp. 45-51.

[11] El-Hamalawi A (2004),A 2D combined advancing front-Delaunay mesh generation scheme, Finite Elements in Analysis and Design, 40(9), 967-989.

[12] Fleischmann P \& Selberherr S (1997),Fully unstructured Delaunay mesh generation using a modified advancing front approach for applications in technology CAD, Journal of Technology Computer Aided Design TCAD,1-38.

[13] Whelan T (1986),A representation of a C2 interpolant over triangles, Computer Aided Geometric Design, 3(1),53-66.

[14] Farrashkhalvat M \& Miles JP (2003),Basic Structured Grid Genera tion: With an introduction to unstructured grid generation. Elsevier.

[15] Abas ZA \& Salleh S (2011), Enhanced Advancing Front Technique with Extension Cases for Initial Triangular Mesh Generation, In Proceedings of the World Congress on Engineering 2011. 